\title{
Estimação de parâmetros genéticos utilizando-se a produção de leite no dia do controle em primeiras lactações de vacas da raça Jersey
}

\author{
Nelson José Laurino Dionello ${ }^{1}$, Carlos Alberto Soares da Silva², Claudio Napolis Costa ${ }^{3}$, \\ Jaime Araújo Cobuci ${ }^{4}$
}

\author{
1 Universidade Federal de Pelotas, Pelotas - RS \\ 2 Doutor pelo PPGZ/FAEM/UFPel, Pelotas - RS. \\ 3 Embrapa Gado de Leite, Coronel Pacheco - MG. \\ ${ }^{4}$ Universidade Federal do Rio Grande do Sul, Porto Alegre - RS
}

RESUMO - Registros da produção de leite de 3.531 controles de 620 primíparas da raça Jersey foram utilizados para a estimação dos componentes de covariância e dos parâmetros genéticos para a produção de leite por meio dos modelos de repetibilidade e de regressão aleatória. O modelo de repetibilidade (MR) incluiu os efeitos fixos de rebanho-ano-mês do controle, estação do parto e idade da vaca ao parto como covariável, com termos linear e quadrático, e os efeitos aleatórios de animal, de ambiente permanente e residual. O modelo de regressão aleatória incluiu os mesmos efeitos do MR, com o uso da função de Wilmink para a modelagem dos efeitos fixo (estação de parto) e aleatórios (genético de animal e de ambiente permanente) As estimativas de variância genética aditiva, de ambiente permanente, residual e fenotípica, e de herdabilidade e repetibilidade para a produção de leite usando-se o MR foram, respectivamente, 7,08; 1,88; 7,79 e 16,76 kg ${ }^{2}$ e 0,42 e 0,50 . Pelo modelo de regressão aleatória, os valores de herdabilidade para a produção de leite decresceram de 0,81 a 0,20 ao longo da lactação As estimativas de variância genética aditiva mantiveram-se praticamente constantes durante a lactação, com maiores valores no início e final da lactação. As correlações genéticas foram próximas à unidade para as produções de leite da fase intermediária da lactação e diminuíram com o aumento do período entre os controles leiteiros. O modelo de repetibilidade não é a melhor alternativa para a modelagem das produções de leite no dia do controle da primeira lactação de vacas Jersey no Brasil.

Palavras-chave: avaliação genética, bovinos de leite, componentes de variância, herdabilidade, regressão aleatória, repetibilidade, seleção

\section{Estimation of genetic parameters for test day milk yield of first lactation Jersey cows using repeatability and random regression models}

\begin{abstract}
Data consisting of 3,531 test day records of 620 first parity Jersey cows were used to estimate genetic parameters for milk yield. Covariance components were estimated by repeatability and random regression animal models. The Wilmink function was used in the random regression model to fit the fixed effect of season of calving and the additive genetic, permanent environment and residual random effects. The repeatability model included the fixed effects of herd-year-month of test, season of calving and age at calving as a covariable with linear and quadratic terms and animal, permanent environmental and residual random effects. The estimates of additive genetic, permanent environmental, residual and phenotypic variances, heritability and repeatability obtained by the repeatability model for milk yield were, respectively: $7.08 ; 1.88 ; 7.79$ and $16.76 \mathrm{~kg}^{2}, 0.42$ and 0.50 . The estimates of heritability for milk yield obtained from the random regression model decreased from 0.81 in the beginning of the lactation to 0.20 in the end. Larger estimates of genetic variance were observed at the extremes of the lactation period, but they were practically constant in mid-lactation. The permanent environmental variances increased along lactation. Genetic correlations between milk yields were larger in close test days, particularly in mid-lactation and decreased for the most distant test days. Genetic parameters do not indicate the repeatability model as an alternative to use test day records in order to estimate breeding values for milk yield of Jersey cows in Brazil.
\end{abstract}

Key Words: dairy cattle, genetic evaluation, heritability, random regression, repeatability, selection, variance components

\section{Introdução}

Tradicionalmente, as informações de produção coletadas no dia do controle leiteiro são acumuladas para constituírem o total da produção na lactação, posterior- mente ajustado para o período de 305 dias, critério padrão para a produção de leite utilizado nas avaliações genéticas de vacas e touros. Nos últimos anos, o ajuste da produção de leite e de seus componentes medidos nos controles leiteiros durante a lactação tem sido proposto como proce- 
dimento alternativo, visando, entre outros objetivos, acelerar o processo de avaliação genética, uma vez que não seria preciso esperar o término da lactação para se obter a informação completa sobre o desempenho dos animais. A utilização da produção de leite no dia do controle como alternativa ao ajuste da produção de leite em 305 dias é feita por meio da metodologia denominada Test Day Models (TDM), pela qual as produções no dia do controle podem ser analisadas sob diferentes pressuposições (Ptak \& Schaeffer, 1993; Swalve, 1995; Swalve, 1998). O TDM mais simples é o modelo de repetibilidade no qual a produção é considerada "característica única" (medidas repetidas do mesmo animal), assumindo-se variâncias homogêneas entre as produções dos controles e correlações genéticas entre elas iguais à unidade, o que, na verdade, acaba não ocorrendo. Igualmente, assume-se que os mesmos genes controlam o desempenho ao longo do tempo. A análise de dados longitudinais por meio dos modelos de regressão aleatória, no entanto, permite considerar variâncias heterogêneas (uma de suas grandes vantagens) entre as produções de controles obtidas ao longo do período da lactação e estimar as diferenças nas curvas de lactação entre animais, essenciais para o estudo da persistência na lactação (Jensen, 2001; Schaeffer, 2004; Cobuci et al., 2004). Confirmando essa idéia, Jamrozik et al. (1997) e Van Der Werf et al. (1998) relataram que os modelos de regressão aleatória são mais flexíveis e precisos que o modelo de repetibilidade ou os modelos que consideram produção total ajustada para 305 dias.

Vários estudos no Brasil (Costa et al., 2002a; Costa et al., 2002b; Melo et al., 2005; Freitas, 2003; Cobuci et al., 2006) têm sido realizados para identificar o melhor modelo de ajuste das produções de leite no dia do controle de diferentes raças, tendo em vista as indicações de que a herdabilidade desta característica é superior à da produção de leite da lactação em 305 dias, o que permitiria maior progresso genético pela seleção (Melo et al., 2001; Melo et al., 2005). Outra vantagem aparente seria o uso de maior número de registros de desempenho por animal, pois os modelos de regressão aleatória não exigem número mínimo de medidas por animal, o que, por sua vez, contribuiria para a maior precisão das estimativas de valor genético dos animais.

Objetivou-se com este estudo estimar parâmetros genéticos em função da produção de leite no dia do controle das primeiras lactações de vacas da raça Jersey utilizando-se os modelos de repetibilidade e de regressão aleatória.

\section{Material e Métodos}

Utilizaram-se 47.024 registros de produção de leite no dia do controle de 4.764 lactações de vacas da raça Jersey. Em virtude do pequeno número de registros observados entre a $2^{\mathrm{a}}$ e a $9^{\mathrm{a}}$ lactação, ao se proceder à estruturação dos dados para as análises, optou-se por usar apenas os registros da primeira lactação. Foram formadas classes de grupo contemporâneo em função do rebanho, ano e mês em que foi realizado o controle leiteiro. Os registros de produção de leite também foram agrupados em quatro estações de parto: janeiro a março, abril a junho, julho a setembro e outubro a dezembro.

Entre os critérios de consistência da base de dados, decidiu-se pelo uso exclusivo dos registros de vacas com idade ao primeiro parto entre 20 e 48 meses, pela eliminação dos registros de produção de leite inferiores a $2,0 \mathrm{~kg}$ ou superiores a $38,0 \mathrm{~kg}$ e pela exclusão dos registros das filhas de reprodutores que não tinham, no mínimo, duas filhas por classe de grupo contemporâneo. Assim, 5.200 registros de 868 lactações e/ou vacas ficaram disponíveis. Posteriormente, observou-se uma freqüência elevada de registros de produção sem a identificação dos pais da vaca $(32,0 \%)$ e muitos rebanhos com poucos controles anuais, o que poderia caracterizar um controle leiteiro seletivo e que resultou em pequeno número de registros por grupo contemporâneo (rebanho-ano-mês do controle). Esses registros foram também eliminados da base de dados, restando para análise 3.531 registros de produção de 620 vacas, filhas de 175 reprodutores, com partos entre 1986 e 2002, em 44 rebanhos, totalizando 1.342 animais na matriz de parentesco.

Para a estimativa dos componentes de (co)variância e dos parâmetros genéticos, utilizaram-se os modelos de repetibilidade e de regressão aleatória. O modelo de repetibilidade foi descrito como:

$$
y_{i j k}=R A M_{i}+E P_{j}+\sum_{n=1}^{2} b_{n} x_{i j k l}+a_{i j k}+p e_{i j k}+e_{i j k}
$$

em que: $y_{i j k}=$ produção de leite no dia do controle; $R A M_{i}=$ efeito do rebanho-ano-mês do controle; $E P_{j}=$ época de parto; $b_{1}$ e $b_{2}=$ coeficientes de regressão para os efeitos linear e quadrático da produção no dia do controle em função da idade $x_{i j k}$ da vaca ao parto em meses; $a_{i j k}=$ efeito genético aditivo da vaca $k$ pertencente ao rebanho-anomês de controle $i$ e à época de parto $j ; p e_{i j k}=$ efeito de ambiente permanente da vaca $k$ pertencente ao rebanhoano-mês de controle $i$ e à época de parto $j$; e $e_{i j k}=$ erro associado a cada observação. 
Para as análises por meio de regressão aleatória, utilizou-se o seguinte modelo:

$y_{i j k l}=R A M_{i}+\sum_{n=1}^{2} b_{n} x_{i j k l}+\sum_{m=1}^{3} \beta_{j m} Z_{k l m}+\sum_{m=1}^{3} a_{k m} Z_{k l m}+\sum_{m=1}^{3} p e_{k m} Z_{k l m}+e_{i j k l}$ em que: $y_{i j k l}$ é a produção de leite no dia do controle; $R A M_{i}$, o efeito fixo do rebanho-ano-mês do controle; $b_{1}$ e $b_{2}$, os coeficientes de regressão para os efeitos linear e quadrático da produção no dia do controle em função da idade da vaca ao parto; $x_{i j k l} ; B_{j m}, a_{k m}, p e_{k m}$, os coeficientes de regressão aleatória para os efeitos fixos e aleatórios genético aditivo e de ambiente permanente, respectivamente, associados aos coeficientes $Z_{k l m}=Z_{t}=\left[\begin{array}{lllll}Z_{k 11} & Z_{k 12} & Z_{k 13}\end{array}\right]^{\prime}=\left[\begin{array}{lll}1 & t & d\end{array}\right]^{\prime}$, $d=\exp ^{-0,05 t}, t$ e $m$ são, respectivamente, número de dias em lactação após o parto e m-ésimo parâmetro da função descrita por Wilmink (1987), utilizada para a modelagem da estrutura de (co)variância entre as produções de leite, tanto da parte fixa (efeito de estação) como da parte aleatória (efeitos de animal e ambiente permanente); e $e_{i j k l}$, o efeito aleatório residual.

A função de Wilmink (1987) é caracterizada por quatro parâmetros e definida por:

$$
y=a_{1}+a_{2} t+a_{3}^{\exp \left(-a_{4} t\right)}
$$

em que $t$ é o número de dias em lactação. Na função de Wilmink, o $4^{\circ}$ parâmetro $\left(a_{4}\right)$ é geralmente considerado uma constante, reduzindo o número de parâmetros a serem estimados de 4 para 3, o que simplifica o ajuste da junção. Neste estudo, assumiu-se o valor de - 0,05 , segundo Wilmink (1987), para o parâmetro $a_{4}$, de forma que $y=a_{1}$ $+a_{2} t+a_{3} \exp ^{(-0,05 t)}$ em que $a_{1}, a_{2}$ e $a_{3}$ estão associados à produção inicial de leite, à taxa de declínio da produção de leite após o pico da lactação e à taxa de ascensão da produção de leite até atingir o pico da lactação, respectivamente. O valor $-0,05$ está relacionado ao estádio da lactação em que ocorre o pico de lactação (exemplo: aproximadamente 50 dias após a data do parto).

Os componentes de (co)variância foram estimados usando-se os programas MTDFREML (Boldman et al., 1995) e REMLF90 (Misztal, 2001), respectivamente, para as análises sob os modelos de repetibilidade e de regressão aleatória.

\section{Resultados e Discussão}

A média da produção diária de leite em controles leiteiros de vacas da raça Jersey com 29,5 meses de idade ao primeiro parto foi $13,86 \pm 5,35 \mathrm{~kg}$. Informações sobre o número de observações, as médias e seus desvios-padrão para a produção de leite e dias em lactação nos controles são apresentados na Tabela 1. Observou-se pequeno aumento da produção, até atingir o máximo, nos controles realizados em média aos 55 dias, que, posteriormente, decresceu paulatinamente até o final da lactação.

As estimativas de variância genética aditiva, de ambiente permanente, residual e fenotípica obtidas utilizando-se o modelo de repetibilidade foram, respectivamente, de 7,08; 1,88; 7,79 e $16,76 \mathrm{~kg}^{2}$. Os valores de herdabilidade e repetibilidade estimados pelo mesmo modelo foram de 0,42 e 0,50 , respectivamente.

$\mathrm{O}$ valor de $7,08 \mathrm{~kg}^{2}$ para a variância genética aditiva encontra-se dentro da amplitude dos valores reportados por Reents et al. (1994), Swalve (1995), Olori (1997), Rekaya et al. (1999), Melo et al. (2005) e Kaya et al. (2003) para a raça Holandesa. No entanto, o valor estimado para a variância de ambiente permanente ficou abaixo da amplitude das estimativas obtidas por esses autores, que variou de 3,26 (Swalve, 1995) a 8,60 (Olori, 1997). A estimativa da variância residual $\left(7,79 \mathrm{~kg}^{2}\right)$ aproximou-se dos valores obtidos por Rekaya et

Tabela 1 - Número de observações (N), médias e desvios-padrão (DP) para dias em lactação e produção de leite no dia do controle Table 1 - Number of records (N), average and standard desviation (SD) for days in milk and respective milk yield by test day

\begin{tabular}{|c|c|c|c|c|c|}
\hline \multirow[b]{2}{*}{$\begin{array}{l}\text { Controle } \\
\text { Test day }\end{array}$} & \multirow[b]{2}{*}{$\begin{array}{c}\mathrm{N}^{\mathrm{o}} \text { observações } \\
\quad \text { N. of records }\end{array}$} & \multicolumn{2}{|c|}{$\begin{array}{c}\text { Dias em lactação } \\
\text { Days in milk }\end{array}$} & \multicolumn{2}{|c|}{$\begin{array}{c}\text { Produção de leite }(\mathrm{kg}) \\
\text { Milk yield }\end{array}$} \\
\hline & & $\begin{array}{l}\text { Média } \\
\text { Average }\end{array}$ & $\begin{array}{c}\text { Desvio-padrão } \\
S D\end{array}$ & $\begin{array}{l}\text { Média } \\
\text { Average }\end{array}$ & $\begin{array}{c}\text { Desvio-padrão } \\
S D\end{array}$ \\
\hline 1 & 498 & 24,15 & 10,30 & 15,60 & 4,90 \\
\hline 2 & 515 & 55,16 & 12,10 & 16,15 & 5,33 \\
\hline 3 & 492 & 86,58 & 13,56 & 15,18 & 5,14 \\
\hline 4 & 465 & 120,09 & 21,54 & 14,10 & 5,19 \\
\hline 5 & 411 & 153,11 & 21,66 & 13,34 & 5,19 \\
\hline 6 & 350 & 182,94 & 21,68 & 12,58 & 5,09 \\
\hline 7 & 310 & 213,01 & 22,03 & 11,82 & 5,03 \\
\hline 8 & 251 & 242,79 & 21,59 & 11,00 & 4,72 \\
\hline 9 & 162 & 263,67 & 23,52 & 10,30 & 3,86 \\
\hline 10 & 77 & 285,07 & 25,25 & 9,90 & 4,03 \\
\hline
\end{tabular}


al. (1999) e Olori (1997) e foi superior às relatadas por Reents et al. (1994) e Swalve (1995).

A estimativa de herdabilidade $(0,42)$ foi próxima das obtidas por Olori (1997) e Melo et al. (2005) e superior às encontradas por Swalve (1995) e Kaya et al. (2003), na raça Holandesa, e Melo et al. (2001), na raça Gir. A estimativa de herdabilidade sugere o modelo de repetibilidade como alternativa de ajuste da produção de leite no dia do controle, haja vista sua maior facilidade computacional e a constatação, no modelo de regressão aleatória, de que as correlações entre as medidas se aproximem de um.

Fatores como população, raça, condições de criação, sistemas de seleção, entre outros, podem determinar diferentes estimativas de herdabilidade para um mesmo caráter. Neste estudo, a estimativa obtida pode ter sido influenciada pelo valor da estimativa da variância de ambiente permanente, que foi muito inferior às encontradas na literatura. Possivelmente, isso também influenciou diretamente o valor obtido para variância fenotípica. Igualmente, a mesma estimativa contribuiu para o valor obtido para a repetibilidade $(0,50)$, o que, na verdade, é um parâmetro populacional, que vai depender da raça estudada, mas pode ser comparada de modo similar ao relatado por Swalve (1995) e inferior às estimativas descritas por Melo et al. (2001) e Melo et al. (2005), de 0,63 e 0,75 , respectivamente. A estimativa de repetibilidade não sustenta a hipótese de que a produção de leite no dia do controle, ao longo da lactação, deva ser considerada uma mesma característica.

$\mathrm{Na}$ Tabela 2 são apresentadas as estimativas de (co)variância dos coeficientes de regressão aleatória para

Tabela 2 - Estimativas de covariâncias dos coeficientes regressão aleatória dos efeitos genéticos e de ambiente permanente para a produção de leite, obtidas pelo ajuste da função de Wilmink

Table 2 - Estimates of genetic and permanent environment covariances of random regression coefficients for milk yield, using the Wilminkfunction

$\hat{G}=\left[\begin{array}{lll}\hat{g}_{11} & \hat{g}_{12} & \hat{g}_{13} \\ \hat{g}_{21} & \hat{g}_{22} & \hat{g}_{23} \\ \hat{g}_{31} & \hat{g}_{32} & \hat{g}_{33}\end{array}\right]=\left[\begin{array}{ccc}19,98 & -0,47 \times 10^{-1} & -8,75 \\ -0,47 \times 10^{-1} & 0,26 \times 10^{-3} & 0,76 \times 10^{-2} \\ -8,75 & 0,76 \times 10^{-2} & 16,42\end{array}\right]$

$\hat{P}=\left[\begin{array}{lll}\hat{p}_{11} & \hat{p}_{12} & \hat{p}_{13} \\ \hat{p}_{21} & \hat{p}_{22} & \hat{p}_{23} \\ \hat{p}_{31} & \hat{p}_{32} & \hat{p}_{33}\end{array}\right]=\left[\begin{array}{ccc}2,19 & -0,92 \times 10^{-2} & -3,57 \\ -0,92 \times 10^{-2} & 0,65 \times 10^{-3} & 0,14 \times 10^{-1} \\ -3,57 & 0,14 \times 10^{-1} & 7,61\end{array}\right]$

Os índices 1, 2 e 3 referem-se aos coeficientes de regressão aleatória $a_{1}$, $a_{2}$ e $a_{3}$ da função de Wilmink.

The indexes 1,2 and 3 refer to random regression coefficients $a_{1}, a_{2}$ and $a_{3}$ of the Wilmink function. os efeitos genéticos e de ambiente permanente, utilizados para o cálculo das variâncias para a produção de leite ao longo da lactação.

As estimativas de herdabilidade, variância genética, ambiente permanente e residual para a produção de leite em determinados dias da lactação são apresentadas na Tabela 3.

As estimativas de variância genética foram mais elevadas no início e final da lactação, com decréscimo no período intermediário. De modo geral, tanto no início como no final da lactação, tem-se menor número de registros de produção de leite, podendo ocorrer dificuldades no ajuste do modelo, que resultam em estimativas mais elevadas nestes períodos. Segundo Cobuci et al. (2005), esse modelo pode ser pouco apropriado para descrever a variância genética dos controles durante o primeiro mês de lactação. Neste sentido, o mesmo poderia ocorrer com as estimativas dos últimos controles da lactação. Comportamento semelhante aos resultados para a produção de leite ao longo da lactação também foi relatado por Jamrozik et al. (1997), Olori (1997), Rekaya et al. (1999) e Cobuci et al. (2005), com a raça Holandesa, e por Freitas (2003), com a raça Girolando.

As estimativas de variância de ambiente permanente (Figura 1) aumentaram ao longo da lactação, seguindo a mesma tendência observada, na raça Holandesa, por Melo et al. (2005), mas diferindo dos resultados obtidos por Freitas (2003), na raça Girolando, na qual as estimativas não apresentaram similar variação durante a lactação.

As estimativas de herdabilidade da produção de leite (Tabela 3) decresceram de 0,81 a 0,20 ao longo do período de lactação (Figura 2). Esta tendência é semelhante à obtida por Costa et al. (2002a) com a raça Gir, mas diverge dos

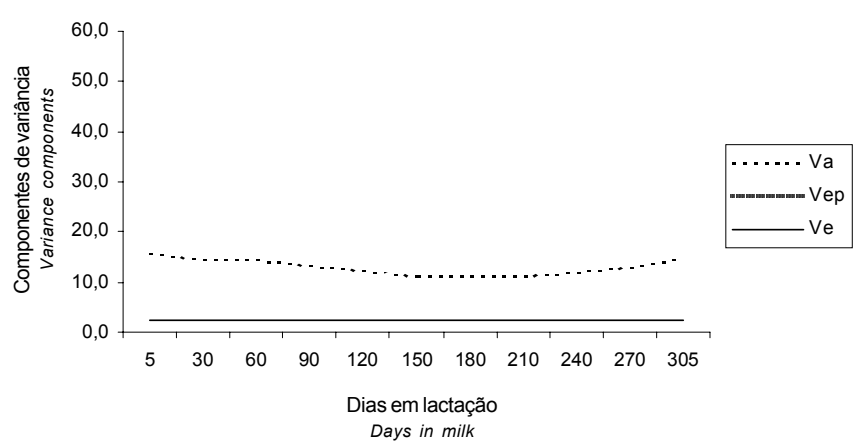

Figura 1 - Variâncias genética $\left(\sigma_{a}^{2}\right)$, de ambiente permanente $\left(\sigma_{e p}^{2}\right)$ e residual $\left(\sigma_{e}^{2}\right)$ ao longo da lactação de vacas Jersey, estimadas por regressão aleatória com a função de Wilmink.

Figure 1 - Genetic $\left(\sigma_{a}^{2}\right)$, permanent environment $\left(\sigma_{e p}{ }^{2}\right)$ and residual $\left(\sigma_{e}{ }^{2}\right)$ variances for milk yield of Jersey cows estimated by random regression using the Wilmink function. 
Tabela 3 - Estimativas de herdabilidade $\left(\mathrm{h}^{2}\right)$, de variâncias genéticas $\left(\sigma_{a}^{2}\right)$, de ambiente permanente $\left(\sigma_{e p}^{2}\right)$ e residual $\left(\sigma_{e}^{2}\right)$ para a produção de leite em dias de lactação de vacas Jersey

Table 3 - Estimates of heritability $\left(h^{2}\right)$, genetic $\left(\sigma_{a}{ }^{2}\right)$, permanent environment $\left(\sigma_{e p}{ }^{2}\right)$ and residual $\left(\sigma_{e}^{2}\right)$ variances of test day milk yield of Jersey cows

\begin{tabular}{lcccc}
\hline $\begin{array}{l}\text { Dias em lactação } \\
\text { Test day }\end{array}$ & $\mathrm{h}^{2}$ & $\sigma_{\mathrm{a}}{ }^{2}$ & $\sigma_{\mathrm{ep}}{ }^{2}$ & $\sigma_{\mathrm{e}}{ }^{2}$ \\
\hline 5 & 0,81 & 15,89 & 1,28 & 2,40 \\
30 & 0,79 & 14,35 & 1,20 & 2,40 \\
60 & 0,72 & 14,38 & 3,17 & 2,40 \\
90 & 0,62 & 13,28 & 5,75 & 2,40 \\
120 & 0,50 & 12,18 & 9,33 & 2,40 \\
150 & 0,41 & 11,44 & 14,05 & 2,40 \\
180 & 0,33 & 11,14 & 19,94 & 2,40 \\
210 & 0,28 & 11,30 & 26,99 & 2,40 \\
240 & 0,24 & 11,92 & 35,23 & 2,40 \\
270 & 0,21 & 13,01 & 44,63 & 2,40 \\
305 & 0,20 & 14,87 & 57,07 & 2,40 \\
\hline
\end{tabular}

resultados relatados por Jamrozik \& Schaeffer (1997), Brotherstone et al. (2000), Cobuci et al. (2004), Freitas (2003) e Melo et al. (2005), para a raça Holandesa, e Guo et al. (2002), para a raça Jersey. A contínua redução nas estimativas de herdabilidade está estritamente associada ao aumento da variância de ambiente permanente que promoveu o aumento da variância fenotípica e à conseqüente redução da contribuição proporcional da variância genética aditiva, que permaneceu praticamente constante ao longo da lactação.

$\mathrm{Na}$ Tabela 4 são apresentadas as estimativas de correlações genéticas e de ambiente permanente para a produção de leite em determinados dias da lactação. De modo geral, as correlações genéticas e de ambiente permanente foram mais altas entre as produções de leite nos dias de controle mais próximos, especialmente na fase intermediária da lactação. Essas correlações reduziram em magnitude para as produções de leite nos dias de controle mais afastados, apresentando, inclusive, valores negativos para as correlações de efeito permanente entre as produções de leite do início da lactação. Essa tendência foi também observada por Olori (1997), Kettunen et al. (2000), Brotherstone et al. (2000), Costa et al. (2002a), Cobuci et al. (2004) e Melo et al. (2005).

Constam na Figura 3 as correlações genéticas aditivas entre as produções de leite nos dias 30,150 e 270 com as de outros dias da lactação. As correlações genéticas entre as produções de controles intermediários e a produção aos 150 dias (C150), entre 60 e 120 dias, aos 30 dias (C30), após os 180 dias e aos 270 dias (C270) apresentaram magnitude superior a 0,90 . As mesmas estimativas entre as produções do controle $\mathrm{C} 150$ e as do período entre 30-240 dias, entre as produções do controle $\mathrm{C} 30$ e as do período até os 150 dias e entre os controles $\mathrm{C} 150$ e C270 foram superiores a 0,80 . A obtenção de correlações genéticas diferentes de 1 comprova a necessidade de se considerarem as medidas repetidas como características diferentes.

Os resultados obtidos neste trabalho podem ter sido influenciados pela estrutura da base de dados, caracterizada pelo baixo número de registros oriundos de poucos rebanhos. Uma alternativa buscada foi a utilização da função de Wilmink, que implica ajuste de menor número de parâmetros que a função de Ali \& Schaeffer (1987) e poderia apresentar ajuste adequado para bases de dados limitadas em número total de registros de produção de leite. Pode-se reportar que, nos estudos iniciais com regressão aleatória, particularmente no Canadá, houve um direcionamento para a adequacidade da função de Wilmink no ajuste da produção de leite e de seus componentes (Jamrozik \& Schaeffer, 1997; Jamrozik et al., 1997), ou mesmo uma ligeira superio-

Tabela 4 - Estimativas de correlações genéticas aditivas (diagonal superior) e de ambiente permanente (diagonal inferior) entre as produções de leite em dias selecionados da lactação de vacas Jersey

Table 4 - Estimates of genetic (above diagonal) and permanent environment (below diagonal) correlations among milk yield in selected days of lactation of Jersey cows

\begin{tabular}{lcccccccccccc}
\hline $\begin{array}{l}\text { DEL } \\
\text { DIM }\end{array}$ & 5 & 30 & 60 & 90 & 120 & 150 & 180 & 210 & 240 & 270 & 305 \\
\hline 5 & & & 0,83 & 0,71 & 0,64 & 0,58 & 0,51 & 0,42 & 0,33 & 0,23 & 0,14 & 0,03 \\
30 & 0,05 & & 0,98 & 0,95 & 0,91 & 0,85 & 0,77 & 0,67 & 0,56 & 0,46 & 0,33 \\
60 & $-0,10$ & 0,95 & & 0,99 & 0,97 & 0,93 & 0,82 & 0,78 & 0,69 & 0,59 & 0,47 \\
90 & $-0,05$ & 0,88 & 0,98 & & 0,99 & 0,97 & 0,92 & 0,85 & 0,77 & 0,68 & 0,99 \\
120 & 0,00 & 0,82 & 0,94 & 0,99 & & 0,99 & 0,96 & 0,91 & 0,81 & 0,77 & 0,68 \\
150 & 0,04 & 0,76 & 0,90 & 0,97 & 1,00 & & 0,99 & 0,96 & 0,91 & 0,85 & 0,77 \\
180 & 0,06 & 0,72 & 0,87 & 0,95 & 0,99 & 1,00 & & 0,99 & 0,96 & 0,91 & 0,85 \\
210 & 0,08 & 0,69 & 0,85 & 0,94 & 0,98 & 0,99 & 1,00 & & 0,99 & 0,96 & 0,92 \\
240 & 0,09 & 0,67 & 0,83 & 0,93 & 0,98 & 0,99 & 1,00 & 1,00 & & 0,99 & 0,97 \\
270 & 0,10 & 0,65 & 0,81 & 0,91 & 0,98 & 0,98 & 0,99 & 1,00 & 1,00 & \\
305 & 0,11 & 0,63 & 0,80 & 0,90 & 0,98 & 0,98 & 0,99 & 1,00 & 1,00 & 1,00 \\
\hline
\end{tabular}

${ }^{1} \mathrm{DEL}=$ dias em lactação $(\mathrm{DIM}=$ Days in milk). 


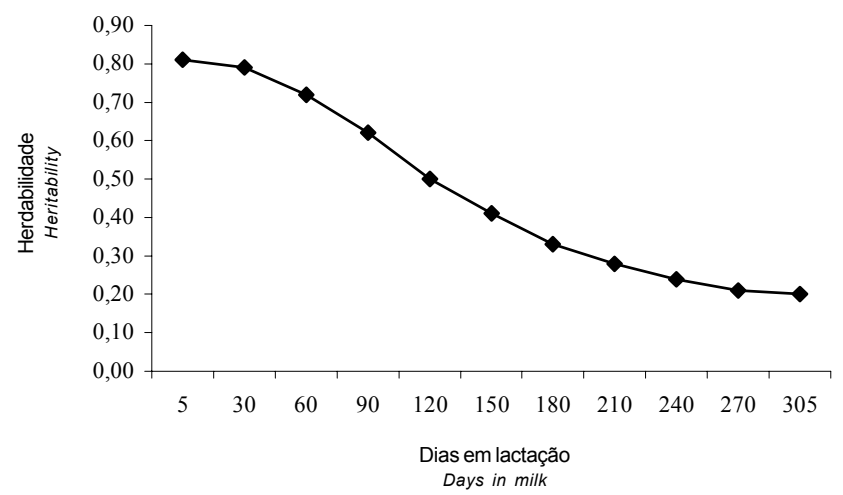

Figura 2 - Herdabilidade da produção de leite nos diferentes dias da lactação de vacas da raça Jersey.

Figure 2 - Heritability of milk yield in different days of lactation of Jersey cows.

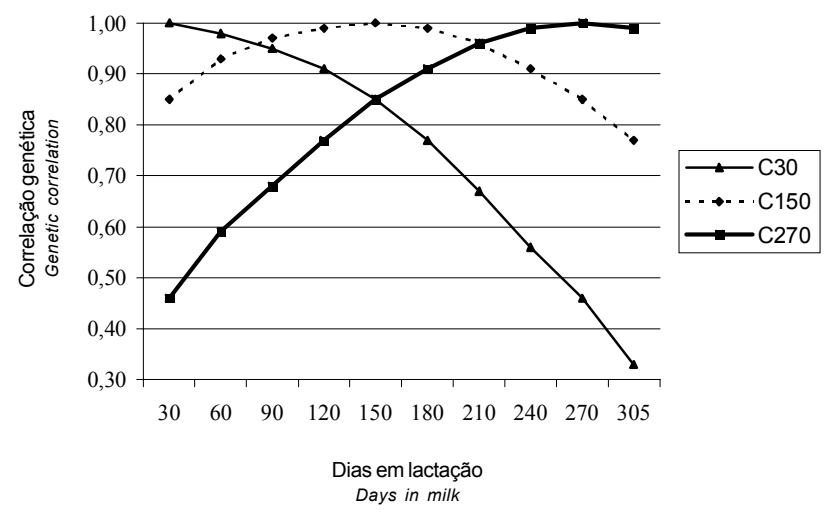

Figura 3 - Correlações genéticas entre as produções de leite nos dias 30, 150 e 270 e as de outros dias da lactação.

Figure 3 - Genetic correlations among milk yield in days 30, 150 and 270 and milk yield in other days of the lactation of Jersey cows.

ridade do uso das funções paramétricas sobre os polinômios ortogonais (Brotherstone et al., 2000). No Brasil, estudos com bases de dados de pequeno tamanho não têm sido muito conclusivos sobre o melhor ou mais adequado ajuste da produção de leite por regressão aleatória com a função de Wilmink (Costa et al., 2002a; Freitas, 2003). Recentemente, Schaeffer (2004) concluiu que os polinômios ortogonais são mais apropriados para ajuste das covariávieis nos modelos de regressão aleatória e que estudos seriam necessários para se identificar a melhor ordem dos polinômios para diferentes aplicações. Considerando-se os resultados encontrados, é importante que os criadores da raça e sua própria associação se conscientizem da importância do serviço de controle leiteiro para efetiva utilização das produções no dia do controle nos programas de seleção para a raça Jersey no Brasil.

\section{Conclusões}

A estimativa de herdabilidade sugere o modelo de repetibilidade como alternativa de ajuste da produção de leite no dia do controle, entretanto, a estimativa de repetibilidade não sustenta a hipótese de que a produção ao longo da lactação deva ser considerada como mesma característica.

O ajuste do modelo de regressão aleatória com uso da função de Wilmink mostrou correlações diferentes da unidade entre as produções de leite ao longo da lactação, confirmando não se tratarem da mesma característica. Adicionalmente, o modelo de regressão aleatória deve ser preferido nos estudos visando estimar parâmetros genéticos na raça Jersey.

Os resultados, em seu conjunto, indicam que novos estudos considerando, inclusive, os polinômios ortogonais devam ser realizados, o que, aliado ao fato de se dispor de uma base de dados com maior número de registros e de animais, poderá proporcionar subsídios para a otimização do processo de seleção na raça Jersey no Brasil.

\section{Agradecimento}

À Associação de Criadores de Gado Jersey do Brasil, à Associação Brasileira de Criadores, à Associação Brasileira de Criadores de Bovinos da Raça Holandesa e à Associação de Criadores de Gado Holandês de Minas Gerais, pela disponibilização dos dados e de outras informações necessárias para a realização deste estudo.

\section{Literatura Citada}

ALI, T.E.; SCHAEFFER, L.R. Accouting for covariances among test day milk yields in dairy cows. Canadian Journal of Animal Science, v.67, p.637-644, 1987.

BOLDMAN, K.G.; KRIESE, L.A.; Van VLECK, L.D. et al. A manual for use of MTDFREML. A set of programs to obtain estimates of variances and covariances. Lincoln: Department of Agriculture. Agriculture Research Service, 1995. 125p.

BROTHERSTONE, S.; WHITE, I.; MEYER, K. Genetic modeling of dairy milk yield using orthogonal polynomial and parametric curves. Animal Science, v.70, p.407-415, 2000.

COBUCI, J.A.; EUCLYDES, R.F.; COSTA, C.N. et al. Análises da persistência na lactação de vacas da raça holandesa, usando produção no dia do controle e modelo de regressão aleatória. Revista Brasileira de Zootecnia, v.33, n.3, p.546-555, 2004.

COBUCI, J.A; EUCLYDES, R.F.; LOPES, P.S. et al. Estimation of genetic parameters for test-day milk yield in Holstein cows using a random regression models. Genetics and Molecular Biology, v.28, n.1, p.75-83, 2005.

COBUCI, J.A.; COSTA, C.N.; TEIXEIRA, N.M. et al. Utilização dos polinômios de Legendre e da função de Wilmink em avaliações genéticas para persistência na lactação de animais da raça holandesa. Arquivo Brasileiro de Medicina Veterinária e Zootecnia, 2006 (no prelo). 
COSTA, C.N.; MELO, C.M.R.; MACHADO, C.H.C. et al. Avaliação das funções polinomiais para o ajuste da produção de leite no dia do controle de primeiras lactações de vacas Gir com modelo de regressão aleatória. In: REUNIÃO DA SOCIEDADE BRASILEIRA DE ZOOTECNIA, 39., 2002, Recife. Anais... Recife. 2002a. (CD-ROM)

COSTA, C.N.; MELO, C.M.R.; MARTINEZ, M.L. et al. Estimation of genetic parameters for test day milk records of first lactation Gir cows in Brazil using random regression (compact disc). In: WORLD CONGRESS OF GENETICS APPLIED LIVESTOCK PRODUCTION, 7., 2002, Montpellier. Proccedings... Montpellier: INRA, Communication n.17-07, 2002b.

FREITAS, M.S. Utilização de modelos de regressão aleatória na avaliação genética de animais da raça Girolando. Viçosa, MG: Universidade Federal de Viçosa, 2003. 78p. Dissertação (Mestrado em Zootecnia) - Universidade Federal de Viçosa, 2003.

GUO, Z.; LUND, M.S.; MADSEN, P. Genetic parameter estimation for milk yield over multiple parities and various lengths of lactation in Danish Jerseys by random regressions models. Journal of Dairy Science, v. 85, n.6, p.1596-1606, 2002.

JAMROZIK, J.; KISTEMAKER, G.J.; DEKKERS, J.C.M. et al. Comparison of possible covariates for use in a random regression model for analyses of test day yields. Journal of Dairy Science, v.80, n.10, p.2550-2556, 1997.

JAMROZIK, J.; SCHAEFFER, L.R. Estimates of genetic parameters for a test day model with random regression for yield traits of first lactation Holstein. Journal of Dairy Science, v.80, n.4, p.762-770, 1997.

JENSEN, J. Genetic evaluation of dairy cattle using test day models. Journal of Dairy Science, v.84, n.12, p.2803-2812, 2001.

KAYA, I.; AKBAS, Y.; UZMAY, C. Estimation of breeding values for dairy cattle using test-day milk yields. Turkey Journal of Veterinary Animal Science, v.27, p.459-464, 2003.

KETTUNEN, A.; MÄNTYSAARI, E.A.; PÖSÖ, J. Estimation of genetic parameters for daily milk yield of primiparous Ayrshire cows by random regression test day models. Livestock Production Science, v.66, p.251-261, 2000.

MELO, C.M.R.; COSTA, C.N.; MARTINEZ, M.L. et al. Parâmetros genéticos para a produção de leite acumulada na lactação e de controles individuais de vacas da raça Gir. In: REUNIÃO DA SOCIEDADE BRASILEIRA DE ZOOTECNIA, 38., 2001. Piracicaba. Anais... Piracicaba: Sociedade Brasileira de Zootecnia, 2001. p.656-657.
MELO, C.M.R.; PACKER, I.U.; COSTA, C.N. et al. Parâmetros genéticos para produção de leite no dia do controle e de primeira lactação de vacas da raça holandesa. Revista Brasileira de Zootecnia, v.34, n.3, p.796-806, 2005.

MISZTAL, I. REMLF90 manual. [2001]. Disponível em: $\mathrm{ftp}: / /$ nce.ads.uga.edu/pub/ignacy/ blupf90/. Acesso em: 10/01/01.

OLORI, V.E. Utilization of daily milk records in genetic evaluation of dairy cattle. Edinburgh, University of Edinburgh, 1997. 230p. Thesis (Ph.D.) - University of Edinburgh, 1997.

PTAK, E.; SCHAEFFER, L.R. Use of test day yields for genetic evaluation of dairy sires and cows. Livestock Production Science, v.34, p.23-24, 1993.

REENTS, R.; DEKKERS, J.C.M.; SCHAEFFER, L.R. Genetic parameters of test day somatic cell counts and production traits. In: WORLD CONGRESS ON GENETICS APPLIED TO LIVESTOCK PRODUCTION, 5., 1994, Guelph. Proccedings... Guelph: University of Guelph, 1994. p.120-123.

REKAYA, R.; CARABAÑO, M.J.; TORO, M.A. Use of test day yields for the genetic evaluation of production traits in HolsteinFrisian cattle. Livestock Production Science, v.57, p.203217, 1999.

SCHAEFFER, L.R. Application of random regression models in animal breeding. Livestock Production Science, v.86, p.34$35,2004$.

SWALVE, H.H. The effect of test day models on the estimation of genetic parameters and breeding values for dairy yield traits. Journal of Dairy Science, v.78, n.4, p.929-938, 1995.

SWALVE, H.H. The use of test day records for genetic evaluation (compact disc). In: WORLD CONGRESS OF GENETICS APPLIED LIVESTOCK PRODUCTION, 1998, Armidale. Proccedings... Armidale: University of New England, 1998. v.23, p.295-301.

Van der WERF, J.H.J.; GODDARD, M.E.; MEYER, K. The use of covariance functions and random regression for genetic evaluation of milk production based on test day records. Journal of Dairy Science, v.81, n.12, p.3300-3008, 1998.

WILMINK, J.B.M. Adjustment of test-day milk, fat and protein yields for age, season and stage of lactation. Livestock Production Science, v.16, p.335-348, 1987.

Recebido: 25/05/05 Aprovado: 15/03/06 